\title{
Australian work health and safety policy for the regulation of psychosocial risks:
}

\section{Perspectives from key informants}

\section{Rachael Elise Potter $^{1 *}$ Valerie O'Keeffe $^{1}$ Stavroula Leka $^{2}$ Maureen Dollard ${ }^{1}$}

\author{
${ }^{1}$ University of South Australia, Adelaide, South Australia, Australia
}

${ }^{2}$ Cork University Business School, Cork, Ireland \& School of Medicine, University of Nottingham, Nottingham, UK

*CONTACT: Rachael Elise Potter, rachael.potter@mymail.unisa.edu.au, University of South Australia, Adelaide, South Australia, Australia

Paper accepted for publication in journal 'Policy and Practice in Health and Safety' on $3^{\text {rd }}$ of March 2019

DOI: 10.1080/14773996.2019.1590765 


\begin{abstract}
The regulation of psychosocial hazards and risks, for the protection of psychological health, is a highly debated issue within work health and safety (WHS). Increasing work-related psychological illness and injury, alongside growing academic evidence and community awareness, has fuelled the need to better prevent and regulate psychosocial hazards and risks. Research must clarify challenges and improvements to policy and practice from stakeholder perspectives. We conduct a qualitative interview-based investigation with 25 informed participants on the effectiveness of Australian WHS policies for the psychosocial risk regulation. Participants are active in diverse roles including policy development, program implementation, industry advice, and psychosocial risk inspection. Inductive analysis revealed divergent viewpoints that are categorised into three broad themes: (1) scant specificity in the current regulatory WHS policy framework, (2) compliance complexities, and (3) the role of regulators in action. Tension points also emerged between these themes and sub-themes, including: (a) how psychosocial risks should be addressed in legislation, (b) how to establish compliance, and (c) the role of the regulator in evaluating compliance, and facilitating education and better practice. Future research must continue to disseminate knowledge from WHS informants to guide better practice. Researchers should investigate organisational barriers that hinder WHS psychosocial risk regulation.
\end{abstract}




\section{Introduction}

The cost of psychological injury compensation claims, greater organisational competition, and increased community awareness have highlighted the need for better protection of workers' psychological health (Lippel \& Quinlan, 2011). Evidence indicates that work health and safety (WHS) policies are far-reaching and powerful strategies that improve organisations' accountability for managing work-related psychosocial risks and psychological health and safety (European Agency for Safety and Health at Work [EU-OSHA], 2012). Legislated WHS policies also empower inspectors and organisational personnel to initiate constructive changes within the workplace. Even in world regions with poor awareness of psychosocial issues, research shows that WHS legislated policies are a key prerequisite for organisational action (Kortum, Leka, \& Cox, 2011). However, within WHS and academic communities, current dialogue around psychosocial risk regulation, and the content and function of WHS policies, is a multifaceted and controversial topic. Despite a great deal of progress, work-related psychological health continues to decline (International Labour Organization [ILO], 2016). This might indicate that current WHS policies have fallen short of the anticipated impact. These outcomes raise critical questions. First, how does the content of WHS policies affect uptake? Second, how can policy translation to practice be improved? Finally, what are the discrepancies or barriers in implementing legislation that need to be addressed to improve policy translation?

Given increases in psychological illness and injury (Safe Work Australia [SWA], 2015, 2018) — and the notable complexities of psychosocial risks (Jespersen, Hasle, \& Nielsen, 2016) — it is vital that WHS policies are continuously evaluated for effectiveness in practice. Few studies have captured the challenges of psychosocial risk regulation through examining the WHS regulatory policy framework as it is perceived by WHS professionals. In practice, these professionals - in consultation with employers - are instrumental in establishing and implementing WHS standards, offering support for compliance and providing advice and guidance (Johnstone, Quinlan, \& McNamara, 2011). In this study we give voice to those who utilise WHS policies during their everyday roles and are active in translating WHS principles into action. We endeavour to convey expertise, experiences and recommendations that have potential to facilitate better practice.

The current study contributes to a broader program of research, supplementing a published paper on Australian WHS policy (Potter, O'Keeffe, Leka, Webber, \& Dollard, 2019). This study mapped Australia's WHS policy context and content, through retrieving all policy instruments relevant to work-related psychosocial risks and the protection of psychological health. It included a gap analysis to determine whether policies reflected principles of good psychosocial risk management. Results showed an inconsistent policy context across Australian jurisdictions, with some jurisdictions being more progressed than others. Further, results indicated poor visibility and specificity of terms in WHS policy content, particularly in the WHS legislation. 
Psychosocial risk regulation and the function of WHS policies are complex societal issues, and qualitative research is needed to generate a deeper understanding. Indeed, policy is not just about the rules but also the way the principles and duties are interpreted and then implemented by stakeholders. The purpose of this study is to extend the previous body of work and determine whether gaps in WHS policy context and content impact regulation (or policy implementation) or if there are further practical regulatory challenges. For those who rely on the policy instruments (i.e., WHS inspectors) does the lack of term visibility noted in Potter et al. (2019) create any barriers for regulation? This study addresses this issue by drawing on the expertise of WHS regulators and other WHS personnel to examine their interpretations of policy content and implementation for psychosocial risk regulation and psychological health protection. But first, we provide an overview of psychosocial risks, followed by a brief summary of their position in policy and then discuss common regulatory approaches undertaken in WHS.

\section{Psychosocial hazards and risks}

Work-related psychosocial hazards are conceptualised as facets of work's design, organisation, and management that arise from the social and organisational environment and have the potential to cause harm (Cox \& Griffiths, 1996). The term psychosocial risk refers to the likelihood for specific psychosocial hazards to cause harm. Recognised psychosocial hazards include excessive workload, high emotional demands, low levels of co-worker support, lack of managerial recognition, and instances of bullying and harassment (Bluff, 2016; Leka \& Cox, 2010). Poorly managed work-related psychosocial hazards are primary contributors of psychological injury and illness (Hall, Dollard, Tuckey, Winefield, \& Thompson, 2010) and can lead to physical injury (Bailey et al., 2015; Li, Dollard, Loerbroks, \& Angerer, 2015). Cumulative evidence shows that psychosocial risk factors contribute to large-scale detrimental effects on organisational productivity (Dollard, Bailey, McLinton et al., 2012) and public health (Nieuwenhuijsen, Bruinvels, \& Frings-Dresen, 2010), predominantly owing to decreased work performance, higher absenteeism rates, and work injury compensation claims (with associated medical costs) (Richards \& Bailey, 2014). The prevalence of psychosocial hazards and risks is increasing and is a feature of developments in the world of work, such as growth in economic globalisation, new communication and information sharing technologies, and a swing towards precarious employment arrangements (Benach et al., 2014; EU-OSHA, 2017). Due to the prominence and severity of poor work-related health, ensuring the prevention, assessment and elimination of psychosocial hazards and risks should be viewed as a priority for regulatory action by WHS inspectors, unions, policy-makers and employers (ILO, 2016).

Yet, the complex and politicised nature of psychosocial risks differentiates them from other work-related risks, making regulation more challenging (Bruhn \& Frick, 2011; Jespersen et al., 2016). Fundamentally, regulation refers to any rule or law endorsed by Government and where there is an 
expectation of compliance (Australian Government, 2017). Unlike physical hazards, psychosocial issues are described as more subjective, contextualised to the individual, workplace, or industry; and linked to multifaceted socio-political changes in the contemporary world of work (Graversgaard, 2004). Likewise, the presence of psychosocial hazards and risks is usually intertwined with managerial powers or ability to design work, distribute resources, and oversee work systems and processes, and to cultivate the nature of the working relationships and climate. Consequently, it is often the structural and organisational mechanisms that generate these hazards and risk factors, which then incites resistance and/or confusion from employers about how to prevent or control psychological health issues (Walters, 2011).

\section{The position of psychosocial risks in WHS policy}

Legislated WHS policies convey a recognised standard and value for workers' health. WHS laws also represent the backbone of regulation, providing a framework to assess organisational compliance with the law and a basis for enforcement and prosecution. Alongside most industrialised countries, Australia sanctioned legislation that obligates employers to identify, assess and control all risks to workers' physical and psychological health as far as reasonably practicable (Bluff, 2016). In addition, soft or non-binding policy instruments such as guidance material and tools have been produced internationally and nationally to assist legislative compliance and good practice (Leka \& Jain, 2016). Recent research by Leka et al. (2015) and Potter et al. (2019) provide comprehensive reviews and analysis of European and Australian WHS policy instruments respectively that are relevant to psychosocial risks. Results from both papers indicate a growing policy context through the development of new policies, yet equally emphasise areas for improvement in terms of content on psychosocial risk management principles.

Additionally, different types of standards are used to frame specific WHS obligations within policies, having implications for how work-related risks are approached in regulation and organisational practice (Bluff, 2017; Bluff \& Gunningham, 2003). These standards consist of specifications, general duties, performance-based and systematic process, or systems-based standards (Bluff \& Gunningham, 2003). Technical or physical risks (e.g., of falls from height) are typically addressed through a command-control approach, whereby the steps for compliance are outlined through specification standards. Conversely, psychosocial risks are often addressed through more reflexive or softer regulation methods, such as general duties, and process- (or systems-) based standards. Australian policy researchers, Bluff and Gunningham (2003), propose that general duties are preferred for psychosocial risks as they allow for a greater amount of flexibility regarding the duty holder's action.

While general duties have certain benefits, thus far, some studies show that the breadth of the legal duties also coincides with considerable uncertainty for employers and WHS inspectors (Lippel, 
Vézina, \& Cox, 2011; Bruhn \& Frick, 2011). In other words, there is often confusion about how to apply the general duties in regulation and practice (Lippel et al., 2011). A prominent study by Johnstone et al. (2011), focusing on generalist inspector attitudes and responses to psychosocial hazards in Australia, revealed that inspectors reported a lack of clarity in the law's provisions, which reduced confidence in prosecuting against noncompliance. In Québec, Lippel et al. (2011) also proposed that the broad legal duties and ambiguous terminology hindered inspectors' initiatives, instigating them to endorse more legislative provisions to mitigate psychosocial risks. In support of these points, a recommendation of the review by Weissbrodt and Giauquie (2017) was to investigate how far it would be possible to outline the legal obligations for psychosocial issues to better assist inspectors and employers (Bruhn \& Frick, 2011; Lippel et al., 2011).

Furthermore, to date, WHS laws around the world have attracted criticism for not referencing the terms psychosocial risk, psychosocial hazard or work-related stress. In particular, European WHS policy research presents critique for the poor clarity and terminology in both hard (i.e., regulatory, legislated) and soft policies (non-legally binding; see Leka et al., 2015; Leka \& Jain, 2016; WiderszalBazyl, Zołnierczyk-Zreda, \& Jain, 2008). The silence on psychosocial terms - and a greater focus on physical risks - negatively impacts prioritisation and management among regulators and workplaces (Ertel et al., 2010; Leka et al., 2011b). European studies establish that half of surveyed stakeholders perceive the current legislation insufficient for psychosocial risk management (Iavicoli et al., 2011; Leka et al., 2011). That said, there still is low prioritisation even where good practice policies have been implemented. Leka and Jain (2016) attribute a lack of action to other contextual hindrances such as poor political will, deregulation, poor social dialogue activity and low levels of resources. Other known issues affecting regulation include a lack of common legislative framework across geographical and political regions, differing levels of inspector training and different health and safety cultures in organisations and regions (Toukas et al., 2015).

\section{WHS state regulators and psychosocial risks}

The relationship between WHS regulators and organisations also shapes regulation. Over time, there has been a shift from state regulation of worker health towards organisational regulated self-regulation (see Black, 2001; Bartle \& Vass, 2007). Self-regulation is characterised as employer's commitment, capability, and undertakings in relation to certain arrangements or processes on an ongoing basis and involves less intervention by state regulators (Bruhn \& Frick, 2011; Walters et al., 2011). In line with the flexibility of the general duties, organisations are now increasingly encouraged to generate their own approaches for risk management and move away from over-reliance on state regulation or methods for compliance (Bluff, 2016). At the same time, these developments mean that psychosocial risk regulation is challenging for inspectors (Rasmussen, Hansen, \& Nielsen, 2011; see Starheim \& Rasmussen, 2014 for overview; Weissbrodt \& Giauque, 2017). To facilitate organisational self- 
regulation, WHS regulators are advised to use a delicate balance of incentives and controls (Starheim \& Rasmussen, 2014). Too much control with an authoritarian focus creates resistance from employers and may hinder the development of good practice. Rather, inspectorates should raise awareness, educate, challenge, and advise companies, developing partnerships outside of formal consultations. This premise is reflected in the Australian National Compliance and Enforcement Policy (SafeWork Australia, 2011), which asserts that regulators should base their service delivery on a mix of encouraging motivation, compliance monitoring and deterrents to drive the uppermost levels of compliance. Indeed, Australian WHS researcher, Johnstone (2004) outlines a proposed enforcement approach, conveying that it is more favourable to begin in an advisory capacity promoting reward and incentives rather than a purely punitive stance. Research from Denmark (Rasmussen, Hansen, \& Nielsen, 2011) and, to a lesser extent, Québec (Lippel, Vézina, \& Cox, 2011) demonstrates that inspectors often undertake a pedagogical position in the organisations they inspect. For this reason, government authorities in various countries have developed more targeted soft law policies and resources to help employers and workers comply with legal duties, comprising voluntary management standards, guides, and practical tools to aid the assessment of psychosocial hazards. Regulators also need high levels of training, expertise and resources for psychosocial risk management.

Further, the regulation of psychosocial hazards and risks is presented as a wicked problem in the academic literature (Jespersen et al., 2016). Wicked problems attract various viewpoints, are highly complex and do not have clear solutions (see Figure 1; Jespersen et al., 2016; Rittel \& Webber, 1973). Wicked problems require a high level of consideration and the involvement of various stakeholders to gain a broader perspective to generate potential resolutions or strategies (Rittel \& Webber, 1973). Understanding the complexity of wicked problems illuminates a clearer path for future policy development and implementation, as well as advancing research methods. Strategies to increase effectiveness in dealing with wicked problems include seeing problems from multiple perspectives, creating a collective learning culture based on collaborative discussion of strategies and engaging many stakeholders in the problem area (Head \& Alford, 2015). 


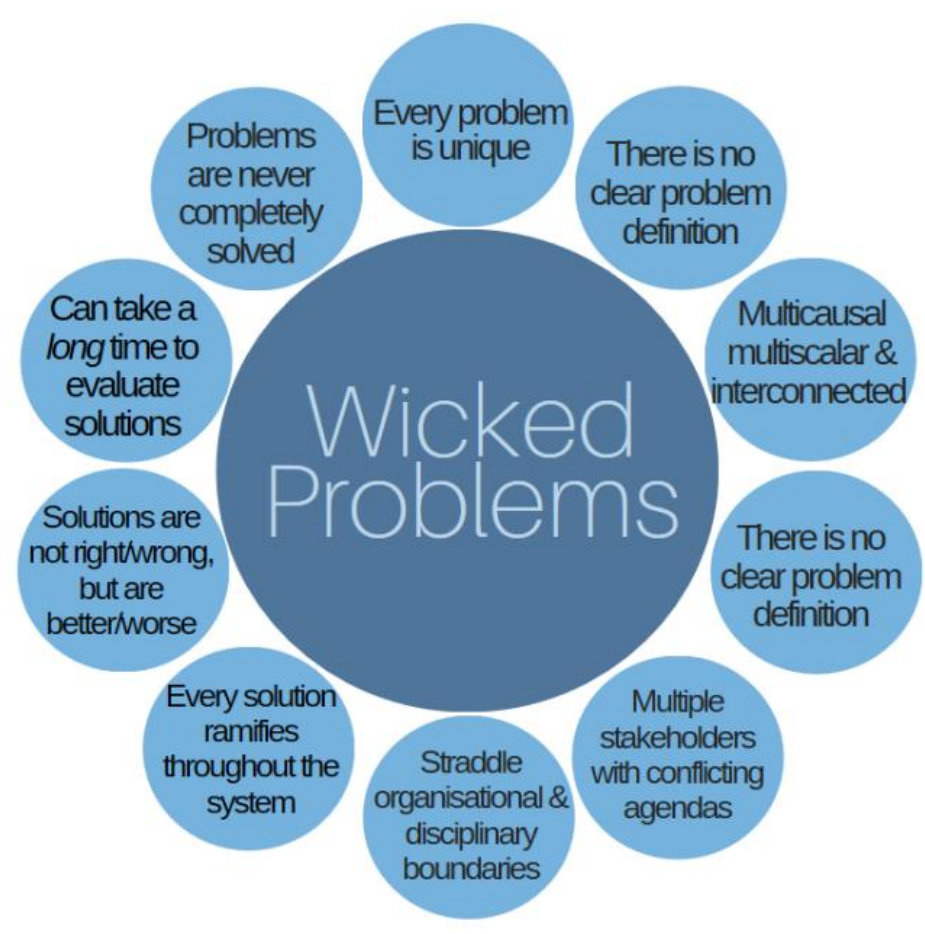

Figure 1. Characteristics of wicked problems (adapted from Rittel \& Webber, 1973).

The regulation of psychosocial risks is a new and under-researched area. A small number of research studies have investigated inspection methods and approaches (see Bruhn \& Frick, 2011; Johnstone et al., 2011; Lippel et al., 2011; Quinlan, 2007; Quinlan, McNamara, \& Johnstone, 2009; Rasmussen, Hansen, Nielsen, 2011; Starheim \& Rasmussen, 2011; Toukas et al., 2015; Velázquez, 2017). Other studies have examined the role of management standards and systems in auditing psychosocial risks within organisations (see Hohnen et al., 2014; Leka et al., 2011 for examples). A number of these regulatory studies are based in Nordic countries and are more focussed on evaluating tools and strategies used by inspectors for the monitoring of the psychosocial work environment. That said, in general, these studies still suggest that developments from a legislative level are needed, together with improvements to inspectorate strategies. Collectively, the researchers make several valuable recommendations for improved practice (see Bruhn \& Frick, 2011), yet none provide specific recommendations to improve policy content in relation to psychosocial hazard and risk regulation. Moreover, despite its context-specificity and social significance, WHS policy has received minimal research attention within countries in the Asia Pacific Region.

\section{The Current Study and Context}

WHS policy serves as the cornerstone for a country's WHS standards and is known to greatly influence organisational practice (Leka \& Jain, 2014). As WHS policy is unique to each country and region, WHS policy research should be extended to beyond Europe, Canada and Nordic countries. To 
date, there is a limited research conducted in Australia and other countries in the Asia Pacific region. There have been three previous studies relevant to psychosocial issues and regulation in Australia (see Johnstone, Quinlan, \& McNamara, 2011; Quinlan, 2007; Quinlan, McNamara, \& Johnstone, 2009). Yet, since the publication of these studies, new WHS policy was implemented in most jurisdictions in 2012 (Johnstone \& Tooma, 2012). There is no research that has since critically evaluated Australian WHS policy relevant to psychosocial issues.

The lack of research on the current policy context, content, and implementation is not sustainable given the increasing national evidence of poor psychological health at work and costly work-related psychological injuries. Expenditure of psychological injury compensation claims has doubled since 2006 and is now estimated to cost AUD543 million per annum (Safe Work Australia, 2018). Research is needed to pinpoint context WHS policy issues and recommendations.

Moreover, over the last five years in Australia there has been a national surge in awarenessraising and prioritisation of psychological health in general. Promoting work-related psychological health is now one of the main targets of the national Australian Work Health and Safety Strategy 2012-2022 (Safe Work Australia, 2012). Moreover, there has been an increased national collaborative effort, evident in the formation of working committees, campaigns, and educational programs. The WHS context has attracted new persons outside the role of generalist WHS inspectors, for example such as specialised psychosocial risk inspectors who have specific training in assessing and managing the presence of psychosocial hazards in targeted groups (Bluff, 2016). However, stakeholder perspectives have been neglected in research. In this study, we aim to determine-from WHS perspectives - whether there can be further tangible developments to policy and practice that will improve regulation and the psychological health of the Australian workforce. Certainly, gaps in policy context and content exist (Potter et al., 2019). Our central aim is to better understand the effectiveness of the WHS regulatory framework (in terms of content and implementation) for psychosocial risks and psychological health. With the awareness that psychosocial risk regulation is a wicked problem, we aim to reveal any major discords or issues that could detract from policy impact.

\section{Method}

\section{Participants}

Qualitative methods are most suitable for uncovering perceptions associated with significant research questions (Eisenhardt \& Graebner, 2007). In line with researcher recommendations (Eisenhardt \& Graebner, 2007; Einsenhardt, Graebner, \& Sonenshein, 2016), we utilise an inductive methodological process best suited for examining themes and patterns in order to understand grand challenges. The current research questions are deemed grand challenges, as national work-related 
psychological health is a significant social issue, necessitating political or policy action to protect psychological health on a large-scale.

Inductive methodological processes depend on theoretical sampling, referring to a selection of cases founded on their capacity to "illuminate and extend relationships among constructs or to develop a deeper knowledge of certain processes" (Einsenhardt et al., 2016, p. 1114). Hence, we sought to interview persons recognised as having a high level of expertise in work-related psychosocial issues. Many participants worked in jurisdictional and/or national positions that involves leadership of larger teams. Participants were contacts who were known to the researchers, via conference meetings and previous work experience, as being highly informed in the area of WHS policy and psychological health regulation. Participants were informed that they could forward the recruitment email on to other persons who they believed might be suitable. Twenty-five participants were involved in this study, producing 20 transcripts. Participants had a broad range of roles and expertise; however, these can be clustered into four main groups of: (1) psychosocial risk inspectors (INSPECT) $(n=7)$, (2) senior WHS union delegates (UNION) $(n=4)$, (3) roles relevant to national and jurisdictional policy-making (POL) $(n=3)$ and (4) personnel in the space of educational or industry projects, programs and industry advisors concerning psychological health (PPI) $(n=6)$. To protect anonymity, the participants' jurisdiction and role is not stated. Participants were senior and leaders in this area.

\section{Procedure}

Data collection occurred between March and September 2017. Potential participants were first contacted via an email approved by the UniSA Human Research Ethics Committee (HREC), which included a participant information sheet and consent terms. Researchers arranged telephone interview times to suit the convenience of the participant, and also provided an option for participants to respond to specific questions via written format. A total of 17 interviews were conducted and two written responses were received. The majority of interviews were conducted individually by the primary researcher, and one interview involved two participants. For one of the written responses, it was reported that six people assisted with the completion of responses. The interviews were conducted by the primary researcher, and one by a secondary researcher, and ranged between 20 minutes to one hour in length. Core questions focussed on the current challenges, the regulatory framework, and the required future focus or changes to improve psychological health at work. All participants were sent a transcript of the interview via email following completion and were able to make changes or clarify any text before sending back to the primary researcher for analysis. 


\section{Analysis}

Interview data were transcribed, inductively coded, and thematically analysed following an inductive analysis process outlined by Thomas (2006). Thematic analysis was used to classify interview text into repeated concepts and phrases. Due to the inductive method, the text was coded without determining categories in advance. Qualitative analysis software NVivo 10 (QRS International, 2018) was used to develop categories, establish themes and highlight relationships relevant to the research questions (Braun \& Clarke, 2006). Procedures were taken to ensure reliability in data analysis. First, consistency checks were frequently conducted with a second researcher. Second, a final draft version of the manuscript was sent to participants, enabling them to provide feedback and validate findings.

\section{Results}

Participants provided descriptive insights into key regulatory challenges and recommendations for policy and practice. Inductive analysis established divergent opinions on the issue of policy content and implementation for the regulation of psychosocial risks. A conceptual map of the key themes, and the relationships among these themes, is presented in Figure 2; as shown, many themes and challenges interrelate and influence one another. Differences in perceptions were evident between the participant 'groups' (e.g., inspectors vs unions) and also within the groups, highlighting that psychosocial risk regulation is a complex topic. Predominantly, the content of policy-and the level of detail and stringency within the WHS regulatory framework-emerged as a tension point within the data. A second key tension point related to compliance and how this is operationalised in organisations. A third point of contention centred on the role of the regulator. There was particular uncertainty and debate over whether regulators should focus on ensuring compliance or on educating organisations about good practice. Participants also described organisational challenges in managing psychosocial risks, but these are beyond the scope of the present discussion. Central themes and subthemes, and the associated relationships and tension points will now be discussed, including illustrative extracts from interviews and transcripts. 


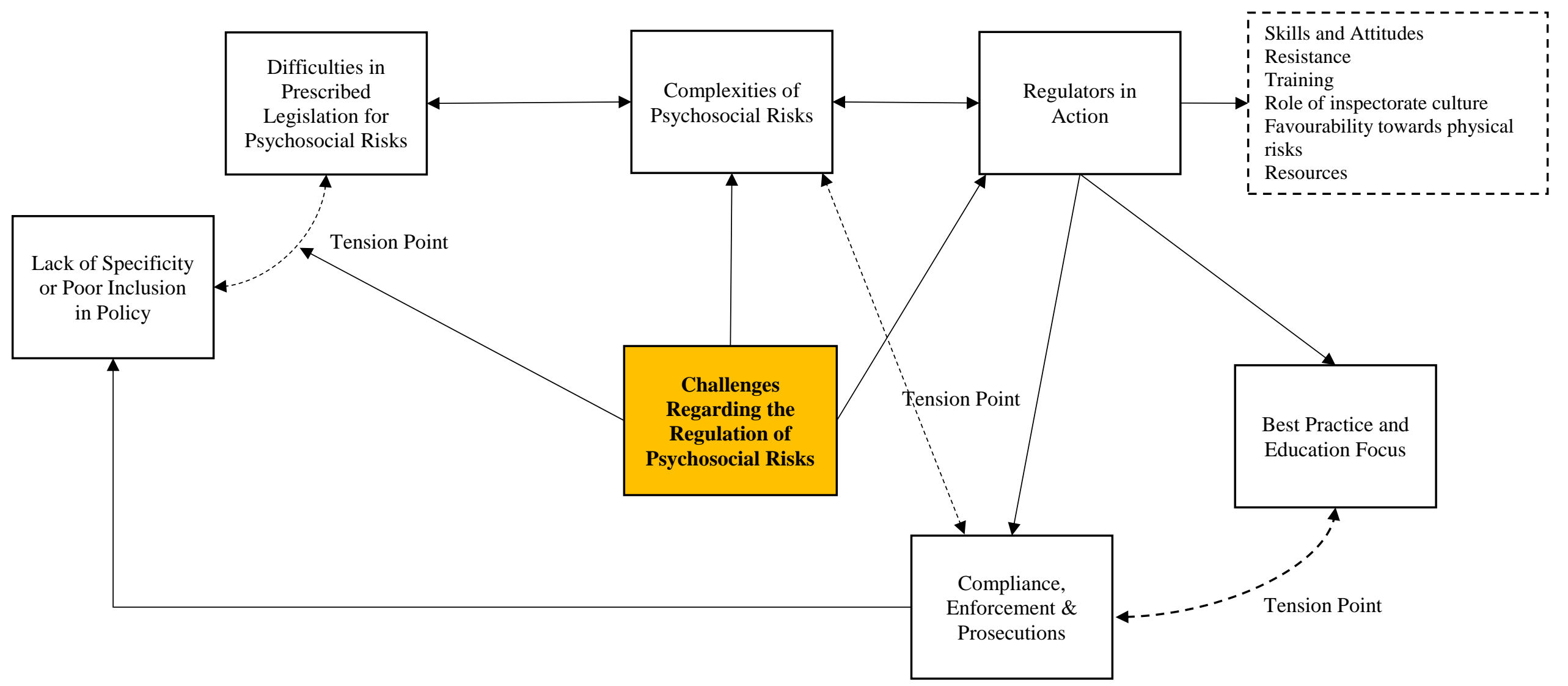

Figure 2. Conceptual map of the themes (and relationships between themes) arising from inductive analysis relevant to the challenges of regulating psychosocial risks 


\section{Lack of specificity in the WHS regulatory framework ("scant specificity")}

Eight participants, comprising two INSPECT, four UNION, one POL and two PPI, had strong views that the current WHS laws are insufficient and have an inadequate level of focus on psychosocial matters. The WHS regulatory framework is integral to regulation, with the WHS Act and Regulations representing the uppermost legislated policy instruments that are referred to during inspection and instances of enforcement or prosecution. The WHS Act establishes the general duties that are placed on various parties involved in the conduct of work (Stewart, 2013). Underpinning the Act, the WHS Regulations stipulate mandatory requirements for duty holders to meet in fulfilling their duty of care, focusing on various issues including worker representation and participation, and managing WHS risks and general workplace arrangements (Stewart, 2013). Following this, there are Codes of Practice (not legally binding, yet admissible in court) and voluntary guidance material.

The view that the current framework is insufficient in content (or lacks specificity) relating to psychosocial hazards and risks is exemplified in the quotation: "To me it's in there because of an addendum. You know "oh and health includes mental health" therefore it's in there" [PP15], and "The current regulatory framework is insufficient in allowing for the inspection and ensuring compliance of protecting psychological health" [INSPECT1]. There was a consensus among these eight participants that the Act and Regulations should be more explicit about on psychosocial risks and psychological health. Specifically, there was a strong call by these eight participants for amendments to the Regulations in order to assist inspectors and regulators in their roles, as is evident in the statement, "work that is hazardous to psychological health could be in a Regulation so that it would actually change the dynamic and would give some ability for regulators and inspectors to be more active around it" [POL3]. However, participants recognised that "getting anything into the Regulations would take quite a journey to determine what should go in and how or what aspects of managing psychological risk will be included" [PPI6]. Moreover, participants made several suggestions about how to amend the WHS regulatory framework, or most optimally, to use the existing general duties during workplace inspection for psychosocial hazards and risks (presented in in Table 1). 
Table 1.

Specific Policy Improvements and/or Use of Policy as Recommended by Participants

\begin{tabular}{|c|c|c|}
\hline Recommendation & Illustrative Quotations & Specific Changes (with Supporting Quotes) \\
\hline $\begin{array}{l}\text { Greater visibility of relevant } \\
\text { terminology (i.e., psychosocial } \\
\text { risk or hazard) within the Act }\end{array}$ & "There should be a greater emphasis on psychological health in the Act". & \\
\hline \multirow{7}{*}{$\begin{array}{l}\text { Inclusion of a Regulation on } \\
\text { psychosocial risks and hazards }\end{array}$} & \multirow{7}{*}{$\begin{array}{l}\text { "Risks that are clearly articulated and prescribed in the Regulations are } \\
\text { regarded as "serious and significant risks". The absence of psychosocial } \\
\text { risks in the regulation sends a particular message to working communities } \\
\text { as to the relative seriousness or significance of these risks". } \\
\text { "You could have a section that expressly talks about psychological health. } \\
\text { What the hazards are and what the risk factors are and that employer must } \\
\text { take reasonably practicable steps to manage it. I think that gives it a } \\
\text { higher elevation - it's a hazard to be managed". }\end{array}$} & \multirow{7}{*}{$\begin{array}{l}\text { Establish Minimum Compliance Standard } \\
\text { "Some dedicated sections of the Regulations around what minimum } \\
\text { compliance looks like or preventing harm and managing psychological } \\
\text { health in the workplace. We can generally communicate what best practice } \\
\text { looks like but we are having trouble saying what compliance looks like". } \\
\text { Risk Assessment } \\
\text { "If I could have an addition to the Regulations that would be something like } \\
\text { employers' have a duty to do a risk assessment of psychological hazards, } \\
\text { because the most difficult part is getting a business to go out and try and } \\
\text { identify their psychological hazards". } \\
\text { Generalisable to All Industry } \\
\text { "...could be generalised enough across all industries. I don't think there's a } \\
\text { need to tailor it to any specific industry sector. For the purpose of policy you } \\
\text { just focus on the minimum". }\end{array}$} \\
\hline & & \\
\hline & & \\
\hline & & \\
\hline & & \\
\hline & & \\
\hline & & \\
\hline $\begin{array}{l}\text { Extending upon or capitalising } \\
\text { on the current sections of 'health } \\
\text { surveillance' in the Regulations } \\
\text { to regulate psychosocial risks }\end{array}$ & $\begin{array}{l}\text { "We may have an opportunity in the area of health surveillance. I } \\
\text { sometimes talk to businesses about exposure to stress being like exposure } \\
\text { to a chemical in the workplace. There might not be effects immediately, } \\
\text { but over time the worker may show the gradual onset of a reaction to the } \\
\text { chemical. With certain hazardous materials, there is a requirement that the } \\
\text { worker has their health monitored. I wonder if there is a role for this in } \\
\text { psychological hazards like stressors". }\end{array}$ & \\
\hline $\begin{array}{l}\text { Clarification around the section } \\
\text { of 'consultation' }\end{array}$ & $\begin{array}{l}\text { "The requirement of consultation is not understood by businesses in the } \\
\text { area of psychological hazards. It is surprising how often checklists and } \\
\text { surveys are used by business and inspectors to identify psychological } \\
\text { hazards, but without a conversation with the workers, and it is their } \\
\text { experience which is the main factor in their stress reaction to a } \\
\text { psychological hazard". }\end{array}$ & \\
\hline
\end{tabular}




\begin{tabular}{|c|c|c|}
\hline Recommendation & Illustrative Quotations & Specific Changes (with Supporting Quotes) \\
\hline $\begin{array}{l}\text { Greater use of Section } 18- \\
\text { 'what is reasonably practicable' }\end{array}$ & $\begin{array}{l}\text { "Unions need to make more use of section } 18 \text {, what's reasonably } \\
\text { practicable. Once you put something to an employer under what is } \\
\text { reasonably practicable - and once you've brought it to their attention } \\
\text { legally- they've got [to show] due diligence on it [to seek information] } \\
\text { and if they ignore it then they are in breach of the law". }\end{array}$ & \\
\hline Due diligence & $\begin{array}{l}\text { "Part of the problem with the law is there is no consultation over due } \\
\text { diligence. That's maybe something that we need to be thinking about for } \\
\text { the next round of reviews, an annual WHS due diligence report for an } \\
\text { employer would lift the veil a bit". }\end{array}$ & \\
\hline $\begin{array}{l}\text { Including research evidence } \\
\text { within the law and specification } \\
\text { of risks }\end{array}$ & $\begin{array}{l}\text { "Other issues arising relate to the quality of evidence, or where known } \\
\text { risks are supported by academia but not that well supported in a } \\
\text { legislative context". }\end{array}$ & $\begin{array}{l}\text { "More research should be put into understanding how you can get evidence } \\
\text { to validate if psychosocial risks are present in the workplace with } \\
\text { confidence. So there's enough research but a group of experts need to get } \\
\text { together and say if these risk factors are present in the workplace it is highly } \\
\text { likely that a psychological injury or illness could result and this could be } \\
\text { used an evidence for an inspector to write an improvement notice to manage } \\
\text { risk". }\end{array}$ \\
\hline \multirow[t]{3}{*}{ Codes of Practice } & \multirow{3}{*}{$\begin{array}{l}\text { "Clearer guidance, preferably in the form of Codes of Practice that have } \\
\text { some legal weight, would be something more tangible for people to pick } \\
\text { up and go ok this is what it means and this is what we have to do. A lot of } \\
\text { them don't know what to do or where to start". }\end{array}$} & $\begin{array}{l}\text { "Bullying is one aspect of psychological health but it is something that you } \\
\text { can do a Code of Practice around". }\end{array}$ \\
\hline & & $\begin{array}{l}\text { "There could be [a Code of Practice] on stress and fatigue management is so } \\
\text { underdone". }\end{array}$ \\
\hline & & $\begin{array}{l}\text { "Codes of Practice around controlling occupational violence and bullying } \\
\text { would be particularly useful". }\end{array}$ \\
\hline \multirow[t]{4}{*}{ Greater guidance and tools } & \multirow{4}{*}{$\begin{array}{l}\text { "The challenge is to provide greater clarity". } \\
\text { "There needs to be a reference point with some consistent guidance for } \\
\text { the employer to have a consistent frame of reference. It's really the actual } \\
\text { tools that help with the doing. The challenge is the how, so getting really } \\
\text { good example of employers that are doing good things, whether it's the } \\
\text { risk management process or undertaking things like surveying and } \\
\text { monitoring the staff's mental health and wellbeing". }\end{array}$} & Focus on Small Businesses \\
\hline & & $\begin{array}{l}\text { "A lot of our businesses fall within [the definition of] a small business and } \\
\text { they just don't have the time or the resources to sit there and work out what } \\
\text { it means for them. So we try and provide examples that are relevant and } \\
\text { where we have tools we try and make sure there's something a small } \\
\text { business can pick up". }\end{array}$ \\
\hline & & Need for Better Implementation \\
\hline & & $\begin{array}{l}\text { "Guidance certainly hasn't been taken further in respect to utilising it more } \\
\text { in workplaces. There's a lack of follow up, it's fine giving information and }\end{array}$ \\
\hline
\end{tabular}


Specific Changes (with Supporting Quotes)

having it there but from there you need to take it to the next step and how it gets applied".

\section{Broader Guidance Materia}

"The guidance material is very much focused on bullying and harassment,

which is only one avenue of stress in the workplace. And one avenue of

psychological harm to workers". 
Other participants acknowledged the broad and general nature of the legislation for psychosocial risk regulation but were not vocal about a lack of specificity. One participant stated that, "the intentions of the broad nature of the legislation indicate that you should be able to pick it [psychosocial hazards] up - the lack of regulation around it makes it a bit harder - but that doesn't mean that the issues can't be tackled" [UNION1]. This point was echoed by an inspector, who had successfully used the current legislation to prosecute employers for non-compliance using the general duties within the Act. However, it was also recognised that "it certainly wasn't without its challenges, but we were one of the first to bring bullying cases to court and to have them successfully prosecuted" [INSPECT5]. Another respondent [INSPECT4] further rationalised how breaches of the law could be put forward using the Act under the general duty of care provision. Yet this participant also acknowledged that issues external to the legislation, such as obtaining witness testimony, so often hindered prosecution and enforcement efforts. To improve the translation of legislative requirements or duties, participants proposed more action in the area of communicating risk controls and disseminating guidance material to organisations. There was strong consensus among participants that more practical and evidence-based tools are needed to disseminate how to translate the legal obligations into organisational action.

Rather than discussing the specificity of legislated policy, two participants (POL1 and POL2) from policy backgrounds argued that the legislation or national regulatory policy framework does not sit in isolation; rather it is surrounded by a greater context of policy strategies that aim to give context and direction. These strategies include the Australian Work Health and Safety Strategy 2012-2022 (SafeWork Australia, 2012b), the Australian Prevention Improvement Framework and worker's compensation and anti-discrimination laws, which also guide the efforts and activities of a range of regulators and cultivate the broader policy environment.

The problem with prescriptive legislation. A prominent subtheme, also relating to the WHS regulatory framework, is the complexity and contention surrounding the ability to prescribe psychosocial risks within the legislation. As a result, several participants stood in opposition to the perspective that the current WHS legislation is not specific enough. Conversely, other participants were clear to state that greater specificity concerning psychosocial risks was still needed in the legislation, yet they simultaneously asserted that highly prescriptive legislation would not be appropriate. One participant stated that for psychosocial risks:

"There might be something somewhere (in the legislation) that we can unpack it a little bit but you couldn't really prescribe it in detail because it's just too subjective. People screaming and yelling at people - that's really clearly unacceptable in a lot of workplaces but if you are in a construction site and you've got a whole lot of noise and cranes and people are at high risk then in some occasions you would quite possibly need to scream and yell at someone to get 
them out of the way so they don't get injured. So you know how would you prescribe that?

You can't really because it would depend, but an unguarded saw is an unguarded saw" [PPI6].

Another inspector acknowledged that the current laws are very general yet was unsure whether legislation could be prescribed within the context of psychosocial risks, stating:

"I don't know if it would be helpful to have something too black and white or prescriptive at the moment. We are trying to move towards cased-based safety being less prescriptive so that we encourage people to continue their thinking rather than following rules. So I don't know if having anything more detailed would be helpful, especially given that we are still trying to figure out what's required" [INSPECT3].

Furthermore, this participant acknowledged that "there's a split" [INSPECT3] among regulators within the WHS context in this view of having more specific legislation or for it to be left in its current form.

Several participants discussed the possibility of implementing a standard similar to the National Standard of Canada for Psychological Health and Safety in the Workplace (Canadian Standards Association, 2013). A proportion of participants saw great benefit in the Canadian standard, with some already implementing its materials and approaches. However, there were also concerns raised about the prescriptive nature of having a psychosocial risk management standard in Australia. This viewpoint was shared by a range of participants, including those who still felt the current Australian legislation should include greater explicitness for psychosocial risks. A central reasoning for not adopting the standard approach is exemplified in the statement:

"From a cultural or a leadership point of view I don't know whether we would be ready.

There would have to be a long campaign of education but I also don't know whether having a list of prescriptive standards would be helpful - unless it's called up in the legislation"

[INSPECT2].

In addition, another participant said that "we probably don't want to go down the standard path because it's very prescriptive in nature However we can learn a lot from establishing what minimum compliance looks like" [PPI4].

6.5.2 Complexities of psychosocial risks and establishing compliance ("compliance complexities").

The multifaceted nature of psychosocial risk and establishing compliance emerged as a critical challenge and somewhat underpins all of the other themes in this study. Regulating psychosocial risks is a complex area, as psychosocial risk factors are not directly observable and also are under employers' prerogative to manage. Psychosocial risks are complex for two main reasons. First, they involve relationships between people and second is the fact that they are related to the work design (job demands). As such, participants identified that regulating psychosocial risks are different 
and more challenging than physical risks. This is exemplified in "it's very easy for something that's a physical tangible object so a trip hazard, plane guard or lighting whatever it is. You look at psychological health and a lot of that comes down to communication, people's behaviour etc" [PPI1]. Several participants also made mention of the importance of considering the complexities of emerging risks such as technology and its impact on work life balance, such as "the boundary between work and family has almost disappeared, bringing home stresses into workplaces and work stresses home through technology such as mobile phones, and notebooks/laptops. Through continuous connection to the workplace via these devices, people are less likely to relax and unwind mentally when away from the workplace" [POL2].

Risk management models. While participants acknowledged that psychosocial risks are complex for organisations and regulators to address, there is a strong level of consensus among participants that within the legislation "the risk management framework applies to psychosocial hazards just like other occupational hazards" [POL1] and "manage the risks like you would any other risk" [PPI6]. A participant with a union background stated "we've been managing physical risks under that same model since the 1970s. So it's not as though we need to teach anyone a new mechanism of risk management - we just need to be able to educate people" [UNION2]. Another participant stated that a future focus needs to be on "gaining working community acceptance on applying the risk management model to psychological risks through educational programs" [INSPECT1]. In general, most participants considered it imperative to extend upon previous knowledge and models rather than reinvent completely new approaches.

The operationalisation of compliance - What does it look like? Establishing how compliance is operationalised in practice was a key issue raised by participants. Participants commented on the practicalities and difficulties in ensuring compliance for the regulation of psychosocial risks due to their complex and subjective nature, "when it comes down to how people communicate, the support [from] day to day that people get, the culture of the organisation-it's much less tangible from an enforcement/compliance perspective how that translates" [PPI1]. However, some respondents reasoned that there are times when psychosocial risks could be more objectively assessed for regulation.

"Simply having workload monitoring systems in place would be monumental. So many times stress claims get accepted because someone's doing twice what the role is meant to be doing. And that's very objective to be able to determine those sorts of things you know there are different measures and it's no less objective than a range of risk management matters" [PPI5]. Another substantial recommendation around operationalising compliance was to ensure that specific risk controls were in place. This is evident in the comment:

"We are exploring well what are the risk control measures in regards to managing that stress, what should the employer have in place to mitigate those risk factors. And it's a big piece of 
work that internally is going to take a long time for us to refine what all those risk control measures are for each industry" [INSPECT4].

Another participant suggested that regulators or organisations should:

"Work backwards from where [the] instance occurred and then determine the systems failures of how risks could have been better managed. A lot of the evidence out there identifies very clearly that the organisational risk factors will have impact of psychological health and wellbeing in the workplace" [PPI3].

Questions were raised about how compliance could be achieved with organisations. First, gaps in knowledge were recognised as a potential barrier towards better outcomes, evident in "there are still some gaps in relation to really having a firm grasp on what psychosocial risks are. How much control can an employer have over that?" [INSPECT5]. There were points made for needing to achieve more clarity around compliance as well as best practice. This is discussed in the statement "there's a need for clarity or that in between what's required and what good practice is" [PPI3]. Participants raised questions such as "what is compliance compared to best practice? Where does it sit on a scale?" [INSPECT4]. Other participants generated suggestions on how inspectors could regulate and ensure compliance more effectively, evident in "a group of experts need to get together and say if these risk factors are present in the workplace it is highly likely that a psychosocial injury or illness could result" [PPI5].

Another challenge relevant to achieving compliance relates to the regulator and the practical difficulties encountered when attempting to enforce constructive change. In this sense, the inspectors sometimes experienced feelings of powerlessness when conducting psychosocial risk regulation. One inspector suggested "the inspectorate at times feels limited in the compliance measures that can be leveraged in this space". The same inspector suggested "assessing, evaluating and improving on the provision of inspectorial compliance tools to secure compliance for psychological risks" [INSPECT1].

Enforcement and prosecutions. Difficulties with enforcement and conducting prosecutions were further raised by participants. In particular, the inspectorate culture (i.e., the jurisdiction itself) was also recognised as a factor influencing compliance, enforcement, and prosecution. This is exemplified in "some states (or jurisdictions), depending on what sort of influences there are politically, tend to use more enforcement action and write more notices than others" [INSPECT2]. Another major contributor to the lack of prosecutions was stated to be the difficulty in obtaining legal evidence, supported in "it's the lack of evidence (that) is really difficult for regulators" [PPI4]. In relation to this point, one inspector commented that "one of the issues with psychological, hazards like bullying is that if it's not documented in emails or text message often you need to start to rely on witness testimony. People can often be confused on the stand or have their own difficulties - they might be poor witnesses or have a different perception" [INSPECT2]. 
Other challenges of enforcement and prosecution related to difficulties associated with the criminal justice system. One participant stated that difficulties in prosecuting arise because: "Our burden of proof is based on the criminal jurisdiction so it's beyond reasonable doubt. Our burden of proof is high in regards to the evidence, so we need a lot of evidence to prove before anything is even before the courts" [INSPECT4]. Another main issue is that the system is focussed on injury rather than being prevention focussed:

"Our system likes to see that there has actually been an injury as a result [of non-compliance], so I know the legislation is risk-based but it is very difficult in the criminal system, and to meet the beyond reasonable doubt standard, to prove risk without there actually being a psychological diagnosis. So without the injury... it doesn't lend itself to prosecuting on a lack of prevention" [INSPECT2].

\section{"Regulators in action"}

A third major theme to emerge within this study was the role of the regulators. Within this theme, there is a point of tension around the regulator's current focus on education versus ensuring compliance. There is a lack of clarity on where the efforts of the regulator should lie. Some participants viewed the strong focus on education (rather than regulation) on psychosocial risks and psychological health as less favourable, whereas other participants viewed the emphasis on education and advice as a more appropriate approach in this context.

Educational or a good practice focus: Beyond compliance ("good practice focus"). Within all the interviews, participants presented a range of extensive educational and collaborative efforts being conducted nationally and at the state level addressing psychological health. It was revealed that the regulators are currently adopting an increased focus on good practice or education of organisations rather than a typical regulatory or compliance-focused approach. There was a great deal of argument from some participants that it is important to aim for a good practice perspective rather than a compliance perspective, such as “... it kind of goes beyond whether it's prosecutable or not because obviously no regulator is looking for minimum compliance although that is the only thing they can prosecute on" [INSPECT5]. This point is further reflected in the following excerpt: "It's about working with workplaces around the importance of leadership, the importance of culture and the importance of supporting staff' [PPI1]. There was support for a strong holistic focus on improving workplace culture and leadership, exemplified in the statement: "the workplace culture makes the difference and a lot of our work is on leadership because leaders and managers are the ones who can really shift the culture" [PPI6]. Furthermore, one participant provided the following reason for addressing psychosocial risks and psychological health with more of an education focus, stating "it's about the culture, the civility, the management support, the leadership commitment" [PPI2].

Additional support for a shift towards education and away from regulation in the traditional sense is exemplified in "it's a good management thing rather than an occupational health and safety (OHS) 
thing because we obviously know enough about what constitutes something that complies with the Act and something that does not comply with the Act in terms of OHS" [INSPECT5]. Also, another participant highlighted that addressing psychosocial risks is evolving and an ongoing learning process, portrayed in "we are kind of all on this learning journey together. Even workplaces with good intent are still fairly unsure of what to do. As I said there's no black and white easy fix in this space. The ultimate purpose is that workplaces are safe for workers. How you achieve that is not always through enforcement, you can achieve that through different avenues. It's a combination of the enforcement, the education, the awareness, the working in partnership together, the investment in the role of leaders' commitment and leadership space. So it has to be the more holistic and sophisticated approach to really address the issue" [PPI2].

However, the education focus of the regulator simultaneously raised a large degree of contention and debate. Many participants stated that regulators are moving away from their regulatory role of ensuring compliance. Some participants expressed frustration and criticism at the regulators' current focus on good practice and education. Union members were particularly vocal that regulators needed to take a stronger compliance-focused approach, rather than education. This point relates to the following subtheme about the regulators' skills and attitudes as a current challenge for effective regulation. One union participant stated that:

"They [the regulators] are all go at the educational level. Education training - which is the lowest order of control. If you actually want to control many of the stressors for people, you'd have more people, staff levels would be better, people wouldn't be doing more with less. There would be action taken at those senior higher levels of control. But they won't. People don't touch that because they see it as their managerial prerogative to screw as much as they can out of people with the least amount of input from them, which is of course counterproductive in the long run" [POL3].

Another participant stated that "we have less people working in a regulatory function that we did ten years ago. We have more activity - but less regulatory work" [PPI5].

Regulator skills and attitudes ("skills and attitudes"). The skills and attitudes of the regulators emerged as a strong subtheme. Regulator skills and attitudes influence the implementation of WHS policy principles. One participant made the comment that:

“Inspectors aren't always capable and willing to address psychosocial risks and so this is one of those areas where inspectors either have an interest in this area or they don't. And like the broader community, some people are more equipped by nature in dealing with distress than others and when I say distress I mean other people's distress. So some inspectors will be more comfortable in dealing with people who are obviously quite distressed in front of them compared to others. So it does limit the response in terms of inspector numbers that we can provide" [INSPECT2]. 
One participant felt that "it's much easier to get the regulator involved in something that is a physical issue than to get them involved in something where people's psychological health is at risk because it's just so much harder to quantify and there's not been as much work done in that area" [UNION3].

Participants from a union background were strongly in agreement that the regulators needed to be more active in regulation and prosecution. As one union participant stated that:

"There's very little that the inspectors will actually dictate needs to happen. Most inspectors from most regulators will go and say have you got a policy on bullying and harassment. If they've got a policy then that's good enough. I could have a policy on guarding all of the machines and it wouldn't make a difference - it doesn't necessarily mean that I implemented that policy. And so therefore the lack of implementation of the policy would indicate that the inspector would be able to say there's noncompliance - they don't look at that. They are scared. They are scared of industrial relations. They are scared whether it will hold up in court so they do a whole lot of stuff about whether they can do it or prosecute if it holds up in court and until recently there has been a total unwillingness to participate in tripartite stuff" [UNION2].

There were calls that the "they [the regulators] are captured. They have been captured by industry" [UNION2]. Yet, there were other participants who commended the skills and efforts of the regulators (when a participant had previously managed a team of psychosocial risk inspectors), stating "it's a tough job and they did it well and they dealt with people well and certainly it's an incredibly tense time. They were well situated to be able to deal with people who were quite fragile, while still doing the job that they were employed to do" [INSPECT5].

Another point to be raised by participants was for regulators to exercise self-care in undertaking the role, as exemplified in the following extracts from two inspectors, both from inspectors (or similar).

"The actual inspectors who do this job we need to be really cognisant of their mental health as well and their psychosocial understanding and the reality is that the demographic of inspectors that I work with are fifty year old men and that's the group that perpetuates the stigma" [INSPECT3]. This point is also reflected in, "one of the most complex issues is dealing with a complainant who is suffering from different mental health issues and we are not psychologists, we are not trained in the sense to provide them the support that they need then. We've dealt with people who are deemed to be high suicide risk and have to talk to their psychologists or GPs (general practitioners). We've even contacted the police to do welfare checks. That's just an added layer to the complexities that we deal with when dealing with the complaints of bullying that come through" [INSPECT4]. 


\section{Discussion and Implications}

We asked Australian WHS informants for insights on policy for psychosocial risk regulation. Conducive with wicked problems, interviews showed that this issue is complex; attracting divergent or fragmented perceptions, as well as differences in ideals and strategic positions (Head \& Alford, 2013, see Figure 1). Participant responses brought forth differing insights and recommendations on how to address work-related psychosocial risks in terms of policy content and practical regulation. Three over-arching themes emerged from the interview data comprising (a), scant specificity of terminology in policy, (b) complexities associated with determining compliance and (c) uncertainty surrounding the role of regulators in action. While participants offered explicit comments on improvements to policy content and implementation (see Table 1), the responses simultaneously highlighted tensions that require further deliberation and clarification to move forward (see Figure 2).

1. The first tension point concerns how far psychosocial risks can be delineated in the legislation.

2. A second complex tension relates to how compliance for psychosocial risk management can be determined and operationalised by regulators.

3. A final tension concerns the role of the regulator, as it shifts from a regulatory (or compliance focused) stance towards an educative position, centred on guiding better practice.

In relation to the first tension point, there was both criticism and debate regarding how best to delineate psychosocial issues in policy (i.e., legislation). Most participants were vocal about the fact that there needs to be greater explicitness and clarity in the WHS Act and Regulations for psychosocial hazards and risks, reinforced by findings in the policy gap analysis (Potter et al., 2019). Moreover, to facilitate good practice, there was strong consensus that greater dissemination of guidance material and practical tools is required to support the legislation. However, for policy content, the strongest call for action related to specifically delineating psychosocial hazards within the WHS Regulations policy instrument, as the current silence on terminology is highly discrepant with the focus on physical hazards. With the absence of a regulation for psychosocial hazards or psychosocial risks, WHS inspectors are required to utilise the broad duties in the WHS Act to initiate or enforce change on these issues. To overcome difficulties associated with applying the general duties, several participants established that the general duties could be capitalised on or better utilised by inspectors to improve regulation (see Table 1). Still, in line with propositions from other policy researchers (cf Birkland, 2005), increasing the visibility of terms in policies is likely to present a clearer message to regulators, employers, and the broader community that work-related psychological health is a valued issue. Indeed, the call for greater clarity was argued to be a means to promote the implementation of current risk management strategies to psychosocial risks and hazards, rather than to 
enable a more command-control approach by regulators. In essence, respondents were clear that greater legislative clarity should not go hand in hand with more prescriptive standards (e.g., specification standards). Despite these points, other participants did not believe that psychosocial hazards could be any further delineated in the legislation (relevant to the second tension point discussed next).

The second key point of tension relates to organisational compliance for psychosocial risk management. For psychosocial hazards, regulators and employers cannot establish distinct threshold values for safe and healthy psychosocial work environments—and again, the general duties are very broad - and this limitation has hindered the effectiveness of traditional methods to regulatory enforcement. As a result, WHS participants advocated for greater articulation of what compliance for psychosocial risk management looks like and how a shared understanding can be created. Compliance is a complex construct and has been previously debated within academic literature (Kagan, Gunningham, \& Thornton, 2011). Elderman and Talesh (2011) argue that compliance is a state that is negotiated by two parties and that both employers and inspectors must make an evaluation of compliance, based on their knowledge of psychosocial risk management and diverse sources of information obtained from the employer (Briner \& Rousseau, 2011). However, further research should unpack whether this could be a suitable approach for psychosocial hazards and risks. Indeed, from the research evidence, it is clear that ensuring compliance within organisations is not a dichotomous or straightforward process.

Due to the current level of confusion surrounding the practicalities of regulating psychosocial risks, participants' responses raised the question as to whether it would even be ethical (or helpful) to pursue a punitive path (i.e., compliance focused). Findings from this study suggest that education and transfer of knowledge about psychosocial risks management processes may be the key. In other literature, Potoski and Prakash (2011 p. 247) argue "punishing benign or unintended non-compliance too severely undermines incentives for firms to take positive steps to comply with Regulations". These points lead to bigger questions about the ethics of aiming for a compliance-level approach at all, and instead whether it would be better to focus on a good or better practice standard. However, some types of psychosocial hazards and risks are able to be regulated and assessed for compliance more objectively; including the implementation of workload monitoring systems and other more objective risk control measures. Overall, as identified from public health campaigns and proposed enforcement approaches (Johnstone, 2004), perhaps it is best to start in an education or awareness raising capacity prior to entering into a compliance focus. Research by Hoel and Einarsen (2010) also emphasises the importance of giving stakeholders time to adapt to concepts promoted by legislation and policy and to translate the principles into practice. After inspectorates become used to implementing policies and duties relevant to psychosocial hazards and risks, a broader approach to 
regulation can emerge, as in Denmark (Rasmussen et al., 2011) where the inspectorate is instructed to inspect all workplaces for a wide range of psychosocial hazards.

Next, conflict emerged around the role of the WHS regulator (and inspector) shifting towards having a more educational than regulatory role. WHS participant groups, especially the union representatives and inspectors, were critical of the fact that regulators have taken a more educational approach, which is not compatible with their role of ensuring compliance in organisations. Certainly, in Australia it is undeniable that there has been a push towards an educational or good practice stance from the regulator that complements the flexible nature of the legislation. It is logical that contention around the role of the regulator is linked to an increasing societal shift towards self-regulated regulation - or more cooperative regulatory approaches (Bluff, 2017) — rather than a stronger focus on insistent regulation by the state. The nature of regulation and the different approaches are prominent debates in WHS (Bluff, 2017), and academic researchers present arguments both for and against selfregulation (see Bartle \& Vass, 2007; Black, 2011). A shift towards greater self-regulation by employers arguably ties in with trends of more deregulation in society that aims to provide organisations with more flexibility and control. Other reasons for the decreased regulatory (i.e., compliance) stance by regulators could be due to the steps outlined in many public health models or promulgation pyramids, whereby education and awareness raising should come before compliance

Yet, this educational approach was not favoured by all participants; rather, there were concerns over the regulator not fulfilling its role of ensuring compliance in organisations. There was some strong criticism of the low level of prosecutions that have occurred, which some inspectors attributed to the difficulty in gathering evidence and the stringency of the criminal system. On the contrary, other WHS informants viewed the educational shift as being more beneficial and highlighted the importance of developing a good culture in the organisation and focusing on contributing aspects such as leadership.

Regardless of the role of the regulators, the lack of inspector resources (e.g., staff numbers) and inconsistent competencies (e.g., skills and attitudes) is a concern. For instance, specialist psychosocial inspectors are only employed in several Australian jurisdictions and in very small numbers. Participants stated that often educational approaches, such as industry workshops, were conducted to increase the reach of assistance from WHS inspectors to employers and overcome limited resources in staff numbers. The findings from this study revealed that the skills and attitudes of WHS regulators and inspectors were inconsistent and, sometimes, less than optimal. Previous research by Graversgaard (2004), an inspector and labour psychologist, has highlighted the need to keep improving the skills and attitudes of psychosocial inspectors to reduce stigma. Other studies also highlight similar findings, including the need for greater inspector competencies such as the ability to handle injured worker emotions (Jespersen et al., 2016; Starheim \& Tasmussen, 2014). Future efforts should be concentrated on ensuring that Australia adopts more consistent approaches to psychosocial 
risk management to ensure that there are adequate numbers of well-trained WHS inspectors who are able to assist in both an educational and compliance-focused capacity.

In addition, the power of stakeholder perception and position was a strong theme throughout this program of research. People form their world view through their experiences, consistent with a social constructionist perspective. Social constructionism proclaims that an individual's reality is decisively shaped by their unique perceptions and experiences (Bakhurst, 2011), formed through interactions within the social environment. Participant responses towards psychosocial risk regulation, and the role of policy, are often moulded through their specific role or position. For instance, those employed in an educational position often perceived educational programs to be most effective in creating change and were therefore less interested in changes to the legislation.

Conversely, those individuals who rely on the legislation (i.e., WHS inspectors) perceived value in increasing the specificity of policy content on psychosocial issues. At the same time, there were some variations in perceptions even within groups (e.g., inspectors) that could be related to other factors such as time spent in job or more individual experiences. The existence of discrepancies between perceptions is consistent with prior European research which found evidence of: (1) a split between the perceived effectiveness of the EU legislation in addressing and managing psychosocial risks (Iavicoli et al., 2011; Leka, Jain, Iavicoli, et al., 2011), (2) noted perceptual disagreements (Leka et al., 2014), and (3) other associated difficulties in achieving consensus (Ertel et al., 2010; Leka, Jain, Iavicoli, et al., 2011).

Since people construct their world view and reality through their own experiences, it is difficult for them to conceive of other ways of understanding a problem (Pronin \& Kugler, 2007). As such, participants in this research exhibited blind spots towards interpretations of other roles. For instance, groups and/or individuals were often highly critical of those in other roles and lacked awareness of the constraints that those roles or persons encountered. Poor insight into others' positions is likely to hinder collaborative efforts in improving national psychological health. Most positive action occurs when there is a united effort with a strong shared vision. Those in WHS positions are encouraged to be introspective and at least aware of other groups' or individuals' perspectives, experiences or constraints—regardless of politics and/or vested interests.

Given the wicked nature of psychosocial risk regulation and policy, the divergent views are not surprising. However, the role of social construction should be a future point of research exploration. Researchers should attempt to unpack social constructionism further to understand its influence on policy development and regulation of psychosocial hazards and risks. For instance, in policy-making, how does social construction impact policy development and content? Can and/or should there be an effort to change or unify perceptions on these issues via intervention research? Also, due to the influence of social construction, policy-making processes must be transparent and enable equal say by all relevant parties. Further, to reduce the wickedness of psychosocial risk 
regulation, nationally consistent tools can be created to guide regulators. While there is no quick fix in addressing wicked problems, there can be greater progress in resolving challenges (e.g., regulation) by generating a larger conversation between stakeholders and building evidence.

Overall, work-related psychological health is gaining considerable government attention in Australia. It is anticipated that findings of the present study will contribute towards this expansive constructive movement through articulating policy and practical suggestions that may improve the translation of legislative requirements and work-related health. As a first point of action, it is recommended that efforts be concentrated on further clarifying the tension points presented within this paper. Since this is an exploratory study, with a relatively small sample, it is advised that future studies continue to clarify these issues in future time points and to gain further perceptions.

\section{Conclusion}

Investigation of regulation of psychosocial risks, with reference to the content and implementation of WHS policy, is an under-researched area. From our analysis, three over-arching themes emerged that related to (a) the scant specificity of relevant terms in the current regulatory WHS policy framework, (b) the challenge of securing compliance for psychosocial risks, (c) and the role of regulators in action. Also, points of tension or conflict emerged within these themes, referring to how psychosocial risks should be addressed in legislation, particularly how to establish compliance, and the role of the regulator. The challenges or tensions points should be used as catalyst for action and conversations within and between WHS regulators. As there is a currently a lack of shared knowledge in this area (EU-OSHA, 2012a; Leka \& Jain, 2016) greater collaboration between academics and policy-makers and WHS regulators is needed to develop an evidence-based body of knowledge that can be widely disseminated and referenced. We also advise that research should consider the implications of social construction and how it affects policy development and psychosocial risk regulation. Research should continue to develop knowledge in the field of psychosocial risk regulation, and also acquire perceptions from organisational personnel on these relevant issues. 


\section{References}

Australian Government. (2017). Cutting Red Tape. The Australian Governments Online Resource for Regulation Reform. Retrieved from: https://www.cuttingredtape.gov.au/handbook/what-dowe-mean-regulation

Bailey, T. S., Dollard, M. F., McLinton, S. S., \& Richards, P. A. (2015). Psychosocial safety climate, psychosocial and physical factors in the aetiology of musculoskeletal disorder symptoms and workplace injury compensation claims. Work \& Stress, 29(2), 190-211.

Bartle, I. \& Vass, P. (2007). Self-regulation within the regulatory state: towards a new regulatory paradigm? Public Administration, 85 (4), 885-905.

Benach, J., Vives, A., Amable, M., Vanroelen, C., Tarafa, G \& Muntaner, C. (2014). Precarious Employment: Understanding an Emerging Social Determinant of Health. Annual Review of Public Health, 35, 229-253.

Birkland, T. A. (2015). An introduction to the policy process: Theories, concepts, and models of public policy making. Routledge.

Black, J. (2001). Decentring regulation: understanding the role of regulation and self-regulation in a 'post-regulatory' world. Current Legal Problems, 54 (1), 103-146

Bluff, E. (2016). The Regulation and Governance of Psychosocial Risks and Work: A Comparative Analysis across Countries. Part 3 Society, Safety and Health. In Global Collaborative Research 2016: Financial Regulation/Society, Safety \& Health/Trade \& Investment. Korea, Korea Legislation Research Institute

Bluff, E. (2017). The regulation of work health and safety. Taken from Regulatory Theory: Foundations and applications (edited by Peter Drahos). Canberra, Australia: ANU Press, the Australian National University.

Bluff, E., \& Gunningham, N. (2004). Principle, process, performance or what? New approaches to OHS standards setting. OHS regulation for a changing world of work, 12-42.

Braun, V \& Clarke, V. (2006). Using thematic analysis in psychology. Qualitative Research in Psychology, 3(2), 77-101.

Briner, R. B., \& Rousseau, D. M. (2011). Evidence-based I-O psychology: Not there yet. Industrial and Organizational Psychology, 4(1), 3-22.

Bruhn, A., \& Frick, K. (2011). Why it was so difficult to develop new methods to inspect work organization and psychosocial risks in Sweden. Safety Science, 49(4), 575-581.

Canadian Standards Association. (2013). Psychological health and safety in the workplace. Prevention, promotion, and guidance to staged implementation, CAN/CSA-Z1003-13/BNQ 9700-803/2013. Canada, Ottawa: Canadian Standards Association. 
Cox, T., \& Griffiths, A. (1996). The assessment of psychosocial hazards at work. In M.J. Schabracq, J.A.M. Winnubst, \& C.L. Cooper (Eds.), Handbook of work and health psychology (pp. 127146). Chichester: Wiley.

Dollard, M., Bailey, T., McLinton, S., Richards, P., McTernan, W., Taylor, A., Bond, S. (2012). The Australian Workplace Barometer: Report on Psychosocial Safety Climate and Worker Health in Australia. Safe Work Australia, Canberra.

Dollard, M.F. \& Bailey, T.S. (2014).The Australian Workplace Barometer: psychosocial safety climate and working conditions in Australia. Australia: Australian Academic Press Group Pty. Ltd, Samford Valley, Queensland.

Edelman, L. B., \& Talesh, S. A. (2011). To comply or not to comply-That isn't the question: How organizations construct the meaning of compliance. Explaining compliance: Business responses to regulation, 103-122.

Eisenhardt, K. M., Graebner, M. E., \& Sonenshein, S. (2016). Grand challenges and inductive methods: Rigor without rigor mortis. Academy of Management Journal, 59(4), 1113-1123.

Eisenhardt, K., \& Graebner, M. (2007). Theory building from cases: Opportunities and Challenges. Academy of Management Journal, 50 (1), 25-32.

Ertel, M., Stilijanow, U., Iavicoli, S., Natali, E., Jain, A., \& Leka, S. (2010). European social dialogue on psychosocial risks at work: Benefits and challenges. European Journal of Industrial Relations, 16(2), 169-183.

European Agency for Safety and Health at Work. (2012). Drivers and Barriers for Psychosocial Risk Management: An Analysis of the Findings of the European Survey of Enterprises on New and Emerging Risks. Luxembourg: Publications Office of the European Union. DOI: http://doi.org/10.2802/92077.

European Agency for Safety and Health at Work. (2017). Second European Survey of Enterprises on New and Emerging Risks (ESENER-2) Overview Report: Managing Safety and Health at Work European Risk Observatory. Luxembourg: EU-OSHA.

Graversgaard, J. (2004). Key role of labour inspection: How to inspect psychosocial problems in the workplace? Stress at work in enlarging Europe. Rome, National Institute for Occupational Safety and Prevention.-2004.-P, 65-76.

Hall, G.B., Dollard, M.F., Tuckey, M.R., Winefield, A.H. \& Thompson, B. (2010). Job demands, work-family conflict, and emotional exhaustion in police officers: A longitudinal test of competing theories, Journal of Occupational \& Organisational Psychology, 83(1), 237-250.

Hoel, H. \& Einarsen, S. (2010). Shortcomings of anti-bullying regulations: the case of Sweden. European Journal of Work and Organizational Psychology, 19, 30-50.

Hohnen, P., Hasle, P., Jespersen, A. H., \& Madsen, C. U. (2014). Hard work in soft regulation: a discussion of the social mechanisms in OHS management standards and possible dilemmas in 
the regulation of psychosocial work environment. Nordic Journal of Working Life Studies, 4(3), 13-30.

Iavicoli, S., Natali, E., Deitinger, P., Rondinone, B.M., Ertel, M., Jain, A., \& Leka, S. (2011). Occupational health and safety policy and psychosocial risks in Europe: The role of stakeholders' perceptions. Health Policy, 101(1), 87-94.

International Labour Organisation. (2016). Workplace stress. A collective challenge. Geneva, Switzerland: International Labour Organisation.

Jespersen, A. H., Hasle, P., \& Nielsen, K. T. (2016). The wicked character of psychosocial risks: implications for regulation. Nordic Journal of Working Life Studies, 6(3), 23.

Johnstone, R. \& Tooma, M. (2012). Work Health and Safety Regulation in Australia: the Model Act. Sydney: the Federation Press.

Johnstone, R., Quinlan, M., \& McNamara, M. (2011). OHS inspectors and psychosocial risk factors: Evidence from Australia. Safety Science, 49(4), 547-557.

Kagan, R. A., Gunningham, N., \& Thornton, D. (2011). Fear, duty, and regulatory compliance: lessons from three research projects. Explaining compliance: Business responses to regulation, 37-58.

Kortum, E., Leka, S., \& Cox, T. (2011). Perceptions of psychosocial hazards, work-related stress and workplace priority risks in developing countries. Journal of occupational health, 53(2), 144155 .

Leka, S., \& Cox, T. (2010). Psychosocial Risk Management at the Workplace Level. In S. Leka \& J. Houdmont (Eds.), Occupational Health Psychology (pp.124-156). Willey-Blackwell.

Leka, S., \& Jain, A. (2016). International Initiatives to Tackle Psychosocial Risks and Promote Mental Health in the Workplace: Is There a Good Balance in Policy and Practice?. In Psychosocial Factors at Work in the Asia Pacific (pp. 23-43). Springer, Cham.

Leka, S., Jain, A., Iavicoli, S., Vartia, M., \& Ertel, M. (2011). The role of policy for the management of psychosocial risks at the workplace in the European Union. Safety Science, 49(1), 558-564.

Li, J., Dollard, M. F., Loerbroks, A., \& Angerer, P. (2015). Cardiovascular disease is associated with the perception of worsening psychosocial work characteristics. International Journal of Cardiology, 186, 149-151.

Lippel, K., \& Quinlan, M. (2011). Regulation of psychosocial risk factors at work: An international overview. Safety Science, 49(4), 543-546.

Lippel, K., Vézina, M., \& Cox, R. (2011). Protection of workers' mental health in Québec: Do general duty clauses allow labour inspectors to do their job?. Safety Science, 49(4), 582-590.

National Mental Health Commission (2014). Mentally Healthy Workplace Alliance. Retrieved from: http://www.mentalhealthcommission.gov.au/our-work/mentally-healthy-workplacealliance.aspx 
Nieuwenhuijsen, K., Bruinvels, D., \& Frings-Dresen, M. (2010). Psychosocial work environment and stress-related disorders, a systematic review. Occupational Medicine, 60(4), 277-286.

Potoski, M., \& Prakash, A. (2011). 11. Voluntary programs, regulatory compliance and the regulation dilemma. Explaining compliance: Business responses to regulation, 245.

Potter, R.E., O'Keeffe, V., Leka, S., Webber, M., \& Dollard, M.F. (2019). Analytical review of the Australian policy context for work-related psychological health and psychosocial risks. Safety Science, 111, 37- 48.

Quinlan, M, Johnstone, R \& McNamara, M. (2009). Australian Health and Safety Inspectors' Perceptions and Actions in Relation to Changed Work Arrangements. Journal of Industrial Relations, 51 (4), 557-573.

Quinlan, M. (2007). Organisational restructuring/downsizing, OHS regulation and worker health and wellbeing. International journal of law and psychiatry, 30(4), 385-399.

Rasmussen, M. B., Hansen, T., \& Nielsen, K. T. (2011). New tools and strategies for the inspection of the psychosocial working environment: The experience of the Danish Working Environment Authority. Safety Science, 49(4), 565-574.

Safe Work Australia (2012). Australian Work Health and Safety Strategy 2012-2022. Retrieved from: https://www.safeworkaustralia.gov.au/system/files/documents/1702/australian-whs-strategy2012-2022.pdf

Safe Work Australia. (2011). National compliance and enforcement policy. Canberra, Australia: Safe Work Australia.

Safe Work Australia. (2015). Work-related mental disorders profile. Canberra, Australia: Safe Work Australia.

Safe Work Australia. (2018). Mental Health. Retrieved from https://www.safeworkaustralia.gov.au/topic/mental-health

Starheim, L \& Bogehus, RM. (2014). Labour Inspection Strategies Addressing the Psychosocial Work Environment. Policy and Practice in Health and Safety, 12(1), 53-70.

Stewart, A., 2013. Stewart's Guide to Employment Law. NSW, Australia: The Federation Press.

Thomas, D. R. (2006). A general inductive approach for analyzing qualitative evaluation data. American journal of evaluation, 27(2), 237-246.

Toukas, D., Delichas, M., Toufekoula, C., \& Spyrouli, A. (2015). The role of labour inspectorates in tackling the psychosocial risks at work in Europe: problems and perspectives. Safety and Health at Work, 6(4), 263-267.

Velázquez, M. (2017). Comparative Study of the Psychosocial Risks Prevention Enforcement by the European Labour Inspectorates. In Psychosocial Risks in Labour and Social Security Law (pp. 31-52). Springer, Cham. 
Walters, D. (2011) Worker representation and psychosocial risks: A problematic relationship? Safety Science 49, pp. 599-606

Weissbrodt, R., \& Giauque, D. (2017). Labour inspections and the prevention of psychosocial risks at work: A realist synthesis. Safety Science, 100, 110-124. 